MATHEMATICS OF COMPUTATION

Volume 66, Number 219, July 1997, Pages 1169-1183

S 0025-5718(97)00872-7

\title{
MODULAR FORMS WHICH BEHAVE LIKE THETA SERIES
}

\author{
K. CHAKRABORTY, A. K. LAL, AND B. RAMAKRISHNAN
}

\begin{abstract}
In this paper, we determine all modular forms of weights $36 \leq k \leq$ $56,4 \mid k$, for the full modular group $S L_{2}(\mathbb{Z})$ which behave like theta series, i.e., which have in their Fourier expansions, the constant term 1 and all other Fourier coefficients are non-negative rational integers. In fact, we give convex regions in $\mathbb{R}^{3}$ (resp. in $\mathbb{R}^{4}$ ) for the cases $k=36,40$ and 44 (resp. for the cases $k=48,52$ and 56). Corresponding to each lattice point in these regions, we get a modular form with the above property. As an application, we determine the possible exceptions of quadratic forms in the respective dimensions.
\end{abstract}

\section{INTRODUCTION}

The famous problem of investigating the number of representations of numbers by positive definite integral quadratic forms is dealt with the use of theta series attached to the corresponding quadratic form. This is one of the classical reasons for the study of modular forms. Following the works of Witt and Kneser, M. Ozeki [5] considered the problem of determining all modular forms in $M_{k}(1)$ (which is the space of modular forms of weight $k$ for the modular group $\left.S L_{2}(\mathbb{Z})\right)(k=12,16)$ which are theta series associated to positive definite, even, integral quadratic forms of $2 k$ variables of determinant unity. Because of the difficulty in attacking this problem for $k \geq 16$ (the cases $k=4,8,12$ being known already, (see [5]), Ozeki [5] considered a weakened form of the problem viz., determining all modular forms in $M_{k}(1)(k=12,16)$ which behave like theta series (in other words, which have constant term unity and all other Fourier coefficients in their $q$-expansions are non-negative rational integers).

The results obtained by Ozeki have been extended for the cases $k=20,24,28,32$ (up to the case $\operatorname{dim} M_{k}(1)=3$ ), using simpler methods, by M. Manickam and B. Ramakrishnan $[2,3]$. These results are presented in the appendix.

Though, in principle, the methods used in $[2,3]$ can be extended to higher dimensional cases, in this paper, we will explicitly solve the problem (getting the convex region determining the modular forms) for the cases $\operatorname{dim} M_{k}(1)=4,5$, i.e., for $k=36,40,44,48,52,56$ using the Simplex method for solving a Linear Programming problem.

We have used Mathematica for our computations. Mathematica is the trade mark of Wolfram Research Inc. We thank Ranjan Srivastava for his help related to Mathematica. We are grateful to the referee for pointing out the applications of our results, which helped us in determining the possible exceptions of integral

Received by the editor May 10, 1995 and, in revised form, October 16, 1995 and March 8, 1996.

1991 Mathematics Subject Classification. Primary 11F11, 11F12, 11F27, $11 \mathrm{~F} 30$.

Key words and phrases. Modular forms, theta series. 
quadratic forms, on the lines of the work done by A. M. Odlyzko and N. J. A. Sloane [4]. We also thank the referee for his suggestions to include the previous results obtained by M. Manickam and the last author.

\section{Preliminaries}

Let $k$ be a natural number. Let us denote by $S_{k}(1)$ the space of cusp forms of weight $k$ for $S L_{2}(\mathbb{Z})$. The following modular forms will mainly be used in our discussion. The first one is the normalized Eisenstein series $E_{k}(z) \in M_{k}(1) \quad(2<$ $k \in 2 \mathbb{Z}$ ) defined by

$$
E_{k}(z)=1+A_{k} \sum_{n=1}^{\infty} \sigma_{k-1}(n) q^{n}
$$

where $q=e^{2 \pi i z}, \quad A_{k}=\frac{-2 k}{B_{k}}$, with $B_{k}$ being the $k$-th Bernoulli number. The other modular form is the well-known cusp form of weight 12 defined by

$$
\Delta(z)=q \prod_{n=1}^{\infty}\left(1-q^{n}\right)^{24}=\sum_{n=1}^{\infty} \tau(n) q^{n},
$$

where $\tau(n)$ is the Ramanujan's function.

\section{The CASES $k=36,40,44$}

In these cases $\operatorname{dim} M_{k}(1)=4$. The space $M_{k}(1)$ is spanned by $E_{k}(z)$ and $f_{i, k}(z), 1 \leq i \leq 3$, where

$$
f_{i, k}(z)=\Delta^{i} E_{k-12 i}(z)=\sum_{n=i}^{\infty} a_{i, k}(n) q^{n}, \quad 1 \leq i \leq 3 .
$$

Let $\phi_{k}(z)=1+\sum_{n=1}^{\infty} a_{k}(n) q^{n} \in M_{k}(1)$ be such that

$$
a_{k}(n) \geq 0 ; \quad a_{k}(n) \in \mathbb{Z} \text { for all } n \geq 1 .
$$

Then our problem is to find all $\phi_{k}(z)$ satisfying (2).

Letting $a_{k}(i)=X_{k, i} \in \mathbb{Z}, 1 \leq i \leq 3$, we get

$$
a_{k}(n)=A_{k} B_{k}(n)+\sum_{i=1}^{3} \alpha_{i, k}(n) X_{k, i},
$$

where $\alpha_{i, k}(n) \in \mathbb{Z}, 1 \leq i \leq 3, n \geq 1$, and $B_{k}(n)$ is defined by

$$
\begin{aligned}
B_{k}(n) & =\sigma_{k-1}(n)-a_{1, k}(n)-\left(\sigma_{k-1}(2)-a_{1, k}(2)\right) a_{2, k}(n) \\
& -\left(\sigma_{k-1}(3)-a_{2, k}(3)-\sigma_{k-1}(2) a_{2, k}(3)+a_{1, k}(2) a_{2, k}(3)\right) a_{3, k}(n) .
\end{aligned}
$$

Since $a_{k}(i)=X_{k, i} \in \mathbb{Z}, 1 \leq i \leq 3$ and $a_{k}(0)=1$, from Lemma A.1 of the appendix, we see that $a_{k}(n) \in \mathbb{Z}$ for all $n \geq 0$. This implies that $A_{k} B_{k}(n) \in \mathbb{Z}$.

Using the Simplex method for solving a Linear Programming problem, from (2) and (3) we get the following bounds for $X_{k, i}$,

$$
0 \leq X_{k, i} \leq I_{k, i}
$$

where $I_{k, i}$ are given in the table below.

\begin{tabular}{|c|c|c|c|}
\hline $\mathrm{k}$ & $I_{k, 1}$ & $I_{k, 2}$ & $I_{k, 3}$ \\
\hline 36 & 43690641 & 1155820295703 & 1694328614487247 \\
40 & 136258892 & 8076880335394 & 16311261365557870 \\
44 & 719376585 & 154458199788843 & 295351327765479655 \\
\hline
\end{tabular}


Let $g_{k}(z) \in S_{k}(1)$ be a normalized Hecke eigenform. Then using the Ramanujan - Petersson estimate proved by Deligne, we have

$$
\left|b_{k}(n)\right| \leq \sqrt{3} n^{k / 2},
$$

where $g_{k}(z)=\sum_{n=1}^{\infty} b_{k}(n) q^{n}$.

Also we know that there exists a basis $\left\{g_{i, k}(z) \mid 1 \leq i \leq 3\right\}$ of $S_{k}(1)$ which are normalized Hecke eigenforms. Now expressing each $f_{i, k}(z)$ as a linear combination of the basis elements $g_{i, k}(z)$ and using (7) we get estimates for the Fourier coefficients $a_{i, k}(n)$ as follows.

$$
\left|a_{i, k}(n)\right| \leq \lambda_{k, i} n^{k / 2},
$$

where

\begin{tabular}{|c|c||c|c||c|c|}
\hline $\mathrm{k}$ & 36 & $\mathrm{k}$ & 40 & $\mathrm{k}$ & 44 \\
\hline$\lambda_{k, 1}$ & 1.732881 & $\lambda_{k, 1}$ & 1.742782 & $\lambda_{k, 1}$ & 1.732488 \\
$\lambda_{k, 2}$ & $8.303377 \times 10^{-6}$ & $\lambda_{k, 2}$ & $3.648166 \times 10^{-6}$ & $\lambda_{k, 2}$ & $9.622303 \times 10^{-7}$ \\
$\lambda_{k, 3}$ & $3.848747 \times 10^{-9}$ & $\lambda_{k, 3}$ & $8.112572 \times 10^{-10}$ & $\lambda_{k, 3}$ & $1.055753 \times 10^{-10}$ \\
\hline
\end{tabular}

Using the bounds from the table (6) and the estimates (8), we can prove that $a_{k}(n) \geq 0$ for all $n$ except for the following cases:

$$
(k, n) \in\{(36,4),(36,6),(36,12) ;(40,5),(40,7),(40,14) ;(44,6),(44,8),(44,16)\} .
$$

We have thus the following theorem.

Theorem 3.1. $a_{k}(n) \geq 0$ if and only if $a_{k}\left(i_{k}\right) \geq 0$ where

$$
i_{k}= \begin{cases}1,2,3,4,6,12 & \text { if } k=36, \\ 1,2,3,5,7,14 & \text { if } k=40, \\ 1,2,3,6,8,16 & \text { if } k=44 .\end{cases}
$$

Using again the Simplex method for solving a Linear Programming problem, we get the following theorem.

Theorem 3.2. $a_{k}\left(i_{k}\right) \geq 0$ and $a_{k}\left(i_{k}\right) \in \mathbb{Z}$ if and only if $X_{k, i}(1 \leq i \leq 3)$ belong to the following convex region in $\mathbb{R}^{3}$ determined by the following vertices :

$$
\begin{aligned}
R_{36}= & \{(0,7.41833,10.84975),(0,7.66048,0),(7.60347,12.06289,0),(7.64038,0,0), \\
& (7.63955,0,13.53880),(0,0,7.93633),(7.57620,11.79038,15.22899),(0,0,0)\} ; \\
R_{40}= & \{(0,7.79332,11.44916),(0,8.13741,0),(8.10535,12.90724,0),(8.13436,0,0), \\
& (8.13372,0,14.61139),(0,0,8.77584),(8.09472,12.54750,16.21248),(0,0,0)\} ; \\
R_{44}= & \{(0,8.23571,12.09800),(0,8.85738,0),(8.77219,14.18881,0),(8.85695,0,0), \\
& (8.85646,0,15.79362),(0,0,9.44320),(8.81136,13.60069,17.47033),(0,0,0)\},
\end{aligned}
$$

where $i_{k}$ is as in Theorem 3.1 and the nonzero entries of the vertices are in logarithm to the base 10 computed till 5 places of decimal.

Remark 3.1. A view of the convex region $R_{44}$ is given in Figure 1. 


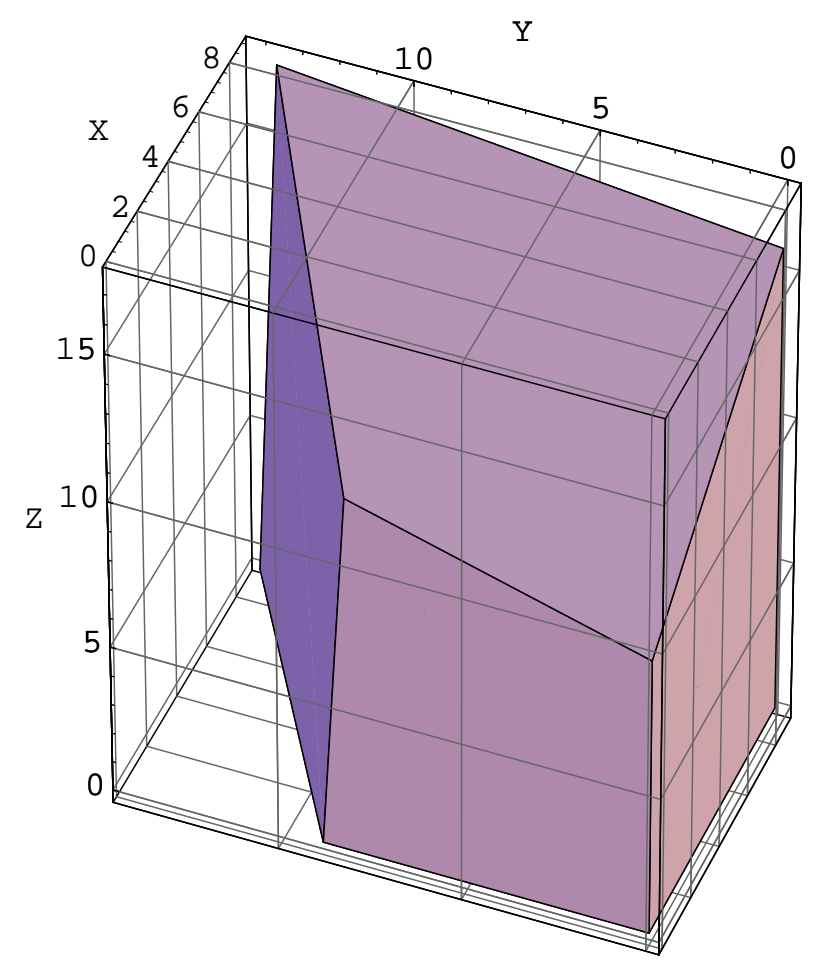

FigURE 1

4. The CASEs $k=48,52,56$

In these cases, $\operatorname{dim} M_{k}(1)=5$. The space $M_{k}(1)$ is spanned by $E_{k}(z)$ and $f_{j, k}(z), 1 \leq j \leq 4$, where

$$
f_{j, k}(z)=\Delta^{j} E_{k-12 j}(z)=\sum_{n=j}^{\infty} a_{j, k}(n) q^{n}, \quad 1 \leq j \leq 4 .
$$

Let

be such that

$$
\phi_{k}(z)=1+\sum_{n=1}^{\infty} a_{k}(n) q^{n} \in M_{k}(1)
$$

$$
a_{k}(n) \geq 0, a_{k}(n) \in \mathbb{Z} \text { for all } n \geq 1 .
$$

The problem is to find all $\phi_{k}(z)$ satisfying (10). Letting $a_{k}(j)=X_{k, j} \in \mathbb{Z}, 1 \leq$ $j \leq 4$, and proceeding as in $\S 3$, we get the following.

The bounds $I_{k, i}$ for $X_{k, i} \quad(1 \leq i \leq 4)$, where $0 \leq X_{k, i} \leq I_{k, i}$ are given in the table below.

\begin{tabular}{|c|c|c|c|}
\hline $\mathrm{k}$ & 48 & 52 & 56 \\
\hline$I_{k, 1}$ & 2686060975 & 10558143244 & 48258047804 \\
$I_{k, 2}$ & 1251190812852642 & 11386858254015760 & 93277927339917074 \\
$I_{k, 3}$ & 14062751051899809150 & 114734698871591508942 & 1439102569361538331277 \\
$I_{k, 4}$ & 12616781321601153192932 & 26896763536380776345441 & 7785250284025588637604758 \\
\hline
\end{tabular}


Theorem 4.1. $a_{k}(n) \geq 0$ if and only if $a_{k}\left(i_{k}\right) \geq 0$, where

$$
i_{k}= \begin{cases}1,2,3,4,5,7,9,10,19, & k=48 \\ 1,2,3,4,6,8,11,12,22, & k=52 \\ 1,2,3,4,7,9,13,26, & k=56\end{cases}
$$

Theorem 4.2. $a_{k}\left(i_{k}\right) \geq 0$ and $a_{k}\left(i_{k}\right) \in \mathbb{Z}$ if and only if $X_{k, j},(1 \leq j \leq 4)$, belong to a convex region, denoted by $R_{k}$, in $\mathbb{R}^{4}$, where $i_{k}$ is as in Theorem 4.1. The regions $R_{k}, 44 \leq k \leq 56$, are given by the following vertices (the nonzero entries of the vertices are given in logarithm to the base 10 till 3 places of decimal).

$$
\begin{aligned}
R_{48}=\{ & (0,0,0,0),(0,0,0,9.77),(0,0,9.843,13.144),(0,9.524,13.682,14.767), \\
& (0,9.521,13.682,0),(0,9.557,0,0),(9.384,0,0,0),(9.384,0,0,17.286), \\
& (9.429,15.097,19.148,22.007),(9.408,14.885,18.782,22.101), \\
& (9.385,0,16.809,0),(9.392,14.979,0,0),(9.392,14.987,0,20.796), \\
& (9.423,14.975,19.138,0),(0,0,10.101,0),(0,9.007,12.902,16.221)\} ; \\
R_{52}= & (0,0,0,10.785),(0,9.68,14.051,0),(0,0,10.596,0),(10.006,0,0,0), \\
& (0,0,10.276,13.785),(0,10.006,0,0),(5.443,11.645,15.681,19.218), \\
& (0,10.184,14.219,17.756),(10.023,16.032,0,0),(10.006,0,0,18.717), \\
& (0,10.031,0,16.404),(10.006,0,17.785,0),(10.006,15.687,20.06,0), \\
& (10.006,0,17.463,20.974),(10.024,16.056,0,22.43),(0,0,0,0)\} ; \\
R_{56}=\{ & (0,0,0,0),(0,0,0,11.615),(0,0,10.664,14.361),(0,0,11.032,0), \\
& (0,10.68,0,17.509),(0,10.684,0,0),(0,10.688,14.875,18.608), \\
& (10.684,0,0,0),(0,10.818,15.395,0),(10.681,16.97,21.158,24.891), \\
& (10.682,16.964,0,23.793),(10.682,16.965,0,0),(10.683,17.103,21.68,0), \\
& (10.684,0,18.409,22.109),(10.684,0,0,20.05),(10.684,0,18.779,0)\} .
\end{aligned}
$$

\section{Applications}

In this section, we use our results of the earlier sections to determine the exceptions of integral quadratic forms following [4]. We use similar notations as in $[4]$.

Let $f_{n}$ denote an even unimodular positive definite quadratic form of dimension $n$. We assume that $n$ is divisible by 8 . Let $T_{n}$ (which denotes the exceptions for $n$ ) be the set of natural numbers $a$ such that some $f_{n}$ does not represent $2 a$. The theta series of $f_{n}$ is

$$
\theta(z)=\sum_{\underline{x}} e^{\pi i f_{n}(\underline{x})}
$$

where the sum extends over all $\underline{x}=\left(x_{1}, x_{2}, \cdots, x_{n}\right)$. Note that $\theta(z) \in M_{n / 2}(1)$. Writing

$$
\theta(z)=\sum_{m=0}^{\infty} a_{n / 2}(m) q^{m}
$$


where $a_{n / 2}(m)$ is the number of times $f_{n}$ represents $2 m$, it is clear that $a_{n / 2}(m) \geq 0$ and we have the famous Witt's bound for $a_{n / 2}(1)$, namely,

$$
a_{n / 2}(1) \leq 2 n(n-1) \text { if } n \geq 16 .
$$

In each dimension $n$, there is a unique theta series having the following Fourier expansion

$$
1+0 \cdot q+0 \cdot q^{2}+\cdots+0 \cdot q^{[n / 24]}+\cdots,
$$

the so-called extremal theta series.

Remark 5.1. Though the cases upto dimension 72 were studied in [4], we have found a mistake in the example given in the paper [4] for the dimension 72 case. Moreover, the referee has pointed out an improvement in the dimension 56 case of [4]. We take this opportunity to thank the referee of the revised version, who has supplied the proof for the improvement of the dimension 56 case, for pointing out the mistakes in our examples (provided for our cases) given in an earlier version of the manuscript. While correcting our examples we have found another mistake in the example of [4] for the dimension 64 case and it turns out that one can get improvement in the dimension 64 case also. We, thus, consider here the cases from dimension 56 to dimension 112.

Remark 5.2. Note that from our method, we get a modular form in $M_{k}(1)$ corresponding to each lattice point in the region $R_{k}(36 \leq k \leq 56)$ which behaves like a theta series. We also note that our method gives the extremal theta series corresponding to the point $(0,0,0)$ (resp. $(0,0,0,0))$ for the case $36 \leq k \leq 44$ (resp. for the case $48 \leq k \leq 56)$.

Remark 5.3. In the following sections we will be determining the possible exceptions of quadratic forms of the respective variable and we do not know whether examples of quadratic forms with those exceptions exist or not.

5.1. Improvement in 56 dimension. The example given in $([4, \mathrm{p} .215])$ corresponds to the point $(810,10434006)$ which is a modular form of weight 28 behaving like theta series (for a proof see the Appendix below). It is interesting to observe that this cannot be a theta series attached to a quadratic form. The proof goes as follows: If a quadratic form represents 2 , then it clearly represents $2^{2} \cdot 2=8$. In other words, the corresponding theta series will have the property that its $q^{4}$-th Fourier coefficient is non-zero whenever its $q$-th Fourier coefficient is non-zero. In this case it has been pointed out in [4] that $a_{28}(1)=0$ and $a_{28}(4)=0$ do not intersect at a lattice point. Thus for 4 to be an exception, one should have $a_{28}(1) \neq 0$, which is impossible as shown above. We, therefore, conclude that

$$
T_{56} \subseteq\{1,2\} .
$$

5.2. Improvement in 64 dimension. The point $(24672,338215905)$ gives the example given in ([4, p.215]) (see Appendix for a proof). Observing the fact that the Fourier coefficients of the theta series (apart from the constant term) attached to a quadratic form are even integers, it will follow that the example cannot be a theta series (the $q^{2}$ coefficient is odd) and above all it violates the theta bound, namely, $a_{32}(1) \leq 8064$. We further observed that $a_{32}(5)=0$ does not have any lattice point inside the theta region (intersection of $R_{32}$, which is described in the Appendix and $\left.a_{32}(1) \leq 8064\right)$. Hence, we have

$$
T_{64} \subseteq\{1,2\} .
$$


5.3. Dimension 72. We have found that the example given in $([4, \mathrm{p} .216])$ for the exceptions 4 and 6 is wrong. In our case, the point $(43659000,714910316160$, 1962733030283070) will provide the above mentioned example and it turns out that the modular form corresponding to this point has a negative Fourier coefficient (12-th), which implies that the above point lies outside the region $R_{36}$. In fact, we have observed that $a_{36}(6)=0$ and $a_{36}(4)=0$ have no intersection within the whole region $R_{36}$. On the other hand, if $a_{36}(6)=0$, then either $a_{36}(1)=0$ or $a_{36}(2)=0$ can happen. The point $(0,36162432,34721684154)$ provides an example which corroborates their result that 6 is a possible exception. The example using the above point is as follows:

$$
\begin{aligned}
1+ & 36162432 q^{2}+34721684154 q^{3}+4528422612000 q^{4} \\
& +370666892907720 q^{5}+2044900385895305412 q^{7} \\
& +213293766887630083440 q^{8}+\cdots .
\end{aligned}
$$

Note that the point $(0,36162432,34721684154)$ lies inside the intersection of $R_{36}$ with $a_{36}(1) \leq 10224$. The intersection region is given in Theorem 5.1.

5.4. Dimension 80. As mentioned earlier, the point $(0,0,0)$ corresponds to the following extremal theta series:

$$
1+1250172000 q^{4}+7541401190400 q^{5}+9236514405888000 q^{6}+\cdots .
$$

Considering the faces of the region $R_{40}$, and using the fact that $a_{40}(1) \leq 12640$, we note that the only planes to be considered are $a_{40}(1) \geq 0, a_{40}(2) \geq 0, a_{40}(3) \geq 0$, $a_{40}(5) \geq 0, a_{40}(7) \geq 0$ and the plane $a_{40}(1) \leq 12640$. Using these planes one gets a bounded region in $\mathbb{R}^{3}$ (which, of course, is a subset of the region $R_{40}$ obtained in Theorem 3.2 which determines the theta series in this case (i.e., for which $a_{40}(1) \leq$ 12640), denoted by $R \theta_{40}$, whose vertices are given in Theorem 5.1.

We also observe that $a_{40}(7)=0$ does not intersect with $R \theta_{40}$ at any lattice point. It seems that there are several lattice points at which $a_{40}(1)=0$ and $a_{40}(5)=0$; $a_{40}(2)=0$ and $a_{40}(5)=0$ intersect. The examples are given below.

The modular form corresponding to the point $(0,3888,614383616)$ is

$$
\begin{aligned}
1+ & 3888 q^{2}+614383616 q^{3}+105073240800 q^{4} \\
& +9485305949851200 q^{6}+3766184235926519808 q^{7}+\cdots
\end{aligned}
$$

and that corresponding to $(32,0,692941200)$ is

$$
\begin{aligned}
& 1+32 q+692941200 q^{3}+118281610976 q^{4} \\
&+ 9971027410950144 q^{6}+3821440153157783200 q^{7}+\cdots .
\end{aligned}
$$

We conclude that

$$
T_{80} \subseteq\{1,2,3,5\} .
$$

5.5. Dimension 88. The point $(0,0,0)$ gives the following extremal theta series:

$$
1+168498000 q^{4}+2480127344640 q^{5}+6298997202432000 q^{6}+\cdots .
$$

The theta bound is $a_{44}(1) \leq 15312$. Like in the previous case, the region $R \theta_{44}$, which determines the theta series is obtained by the following planes $a_{44}(1) \geq 0$, 
$a_{44}(2) \geq 0, a_{44}(3) \geq 0, a_{44}(6) \geq 0, a_{44}(8) \geq 0$ and the plane $a_{44}(1) \leq 15312$. The region $R \theta_{44}$ in $\mathbb{R}^{3}$ is described by its vertices in Theorem 5.1

Though $a_{44}(8)=0$ is involved in determining the theta region, it does not intersect with any lattice point in the region. On the other hand, $a_{44}(1)=0$ and $a_{44}(6)=0 ; a_{44}(2)=0$ and $a_{44}(6)=0$ have intersections with (possibly) many lattice points. We will give some examples below.

The modular form corresponding to the point $(0,235008,4482334086)$ is

$$
\begin{aligned}
& 1+235008 q^{2}+4482334086 q^{3}+1843123327200 q^{4} \\
& \quad+156784934734200 q^{5}+5163935354086047228 q^{7}+\cdots
\end{aligned}
$$

and that corresponding to the point $(1284,0,13884968796)$ is

$$
\begin{aligned}
& 1+1284 q+13884968796 q^{3}+5670311737200 q^{4} \\
& \quad+444535383648744 q^{5}+12234602633705590296 q^{7}+\cdots .
\end{aligned}
$$

We can now conclude that

$$
T_{88} \subseteq\{1,2,3,6\} .
$$

In the following theorem, we give the vertices of the theta regions for the above three cases.

Theorem 5.1. The vertices of the theta regions $R \theta_{k}$, for $k=36,40,44$ are given below (till 4 places of decimal).

$$
\begin{aligned}
R \theta_{36}=\{(0,0,0),(0,0,86363550),(0,45760226.5708,0),(10224,0,0), & (0,26202334.8495,70754060639.13),(10224,0,8193582046), \\
& (10224,340217404.1854,0),(10224,193612838.52,530367407534.3437)\} ; \\
R \theta_{40}=\{(0,0,0),(0,0,596818707.6923),(0,137219474.3535,0),(12640,0,0), & (12640,938218697.7658,0),(12640,0,38565203169.2308), \\
& (0,62133345.6593,281298083974.2577), \\
& (12640,420655108.6645,1938968601537.1154)\} ; \\
R \theta_{44}=\{(0,0,0),(0,0,2774634395.8051),(0,720080843.2055,0),(15312,0,0), & (15312,4716227066.9582,0),(15312,0,135267967990.6522), \\
& (0,172072043.2049,1253146429706.0514), \\
& (15312,1114742540.0899,8235611319530.06)\} .
\end{aligned}
$$

Remark 5.4. A view of the theta region $R \theta_{44}$ (where the nonzero entries are scaled to logarithms to the base 10) is given in Figure 2.

5.6. Dimension 96. As mentioned earlier the point $(0,0,0,0)$ corresponds to the extremal theta series and is given by

$$
1+565866362880 q^{5}+2972108280960000 q^{6}+4164608980546560000 q^{7}+\cdots .
$$

Examining the region $R_{48}$ together with the fact that $a_{48}(1) \leq 18240$, it follows that the only hyperplanes to be considered are $a_{48}(i) \geq 0, i=1,2,3,4,5,7,9,10$. Of these, $a_{48}(9)=0$ and $a_{48}(10)=0$ have no integer solutions for which $a_{48}(1) \leq$ 18240 holds. The examples for the exceptions 5 and 7 are given by the points 


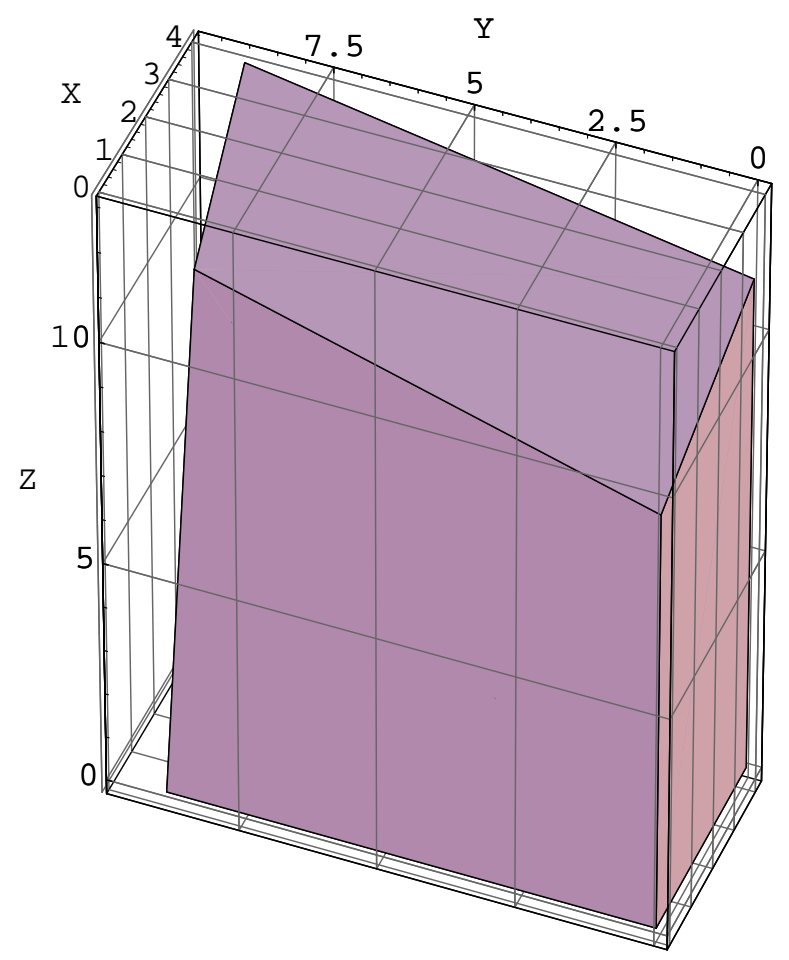

Figure 2

$(0,0,0,5894441280)$ and $(0,139216,14628704256,0)$ respectively. The corresponding modular forms are given as follows.

(24)

$$
\begin{gathered}
1+5894441280 q^{4}+2998421066833920 q^{6}+4163820540080947200 q^{7} \\
+2214108303283082606400 q^{8} \cdots,
\end{gathered}
$$

$$
\begin{aligned}
& 1+139216 q^{2}+14628704256 q^{3}+2819801455167488 q^{5}+50313952102979520 q^{6} \\
& +2340778665780864018752 q^{8}+\cdots .
\end{aligned}
$$

Thus we can conclude that

$$
T_{96} \subseteq\{1,2,3,4,5,7\} .
$$

5.7. Dimension 104. First we will give the extremal theta series corresponding to the point $(0,0,0,0)$ given by

$$
1+91508901120 q^{5}+1000989033408000 q^{6}+2598412027506048000 q^{7}+\cdots .
$$

In this case the intersection of $R_{52}$ with $a_{52}(1) \leq 21424$, which is the theta bound, is given by the hyperplanes $a_{52}(i) \geq 0$ for $i=1,2,3,4,6,8,11$ and $a_{52}(1) \leq 21424$. It turns out that $a_{52}(8)=0$ has no integer points within the above intersection. On 
the other hand, $a_{52}(6)=0$ and $a_{52}(11)=0$ meet both $a_{52}(1)=0$ and $a_{52}(3)=0$. The points

$$
(0,176,0,61392071210) \text { and }(0,10226341170,0,3421557772412480)
$$

provide the above examples which are given as follows:

$$
\begin{aligned}
1+ & 176 q^{2}+61392071210 q^{4}+8936030463136 q^{5}+2646041231718049664 q^{7} \\
& +2355670193686679378944 q^{8}+\cdots
\end{aligned}
$$

$$
\begin{aligned}
& 1+10226341170 q^{2}+3421557772412480 q^{4}+728799703895900160 q^{5} \\
& +340289802570745622520 q^{6}+141143564585883328839680 q^{7} \\
& +14278746426172898992300080 q^{8}+513768402318552455404584960 q^{9} \\
& +5632618326414854488471195420 q^{10}+1659754425886884680776487919360 q^{12} \\
& +\cdots
\end{aligned}
$$

Thus we have established the following

$$
T_{104} \subseteq\{1,2,3,4,6,11\} .
$$

5.8. Dimension 112. In this case the theta bound is $a_{56}(1) \leq 24864$. The intersection of $R_{56}$ with the above theta bound is determined by the hyperplanes $a_{56}(i) \geq 0$ for $i=1,2,3,4,7,9,13$ and $a_{56}(1) \leq 24864$. Surprisingly $a_{56}(i)=0$ does not contain any integer solution within the above intersection for $i=7,9$ and 13 . However, the extremal theta series corresponding to the point $(0,0,0,0)$ is given by the following

$$
\begin{aligned}
1+ & 10888335360 q^{5}+247006775232000 q^{6}+1187911731935232000 q^{7} \\
+ & 1837981772066610324000 q^{8}+1195996575532999166976000 q^{9}+\cdots
\end{aligned}
$$

and hence we conclude that

$$
T_{112} \subseteq\{1,2,3,4\}
$$

For the cases $48 \leq k \leq 56$, the intersection of the region $R_{k}$ and the theta bound $a_{k}(1) \leq 4 k(2 k-1)$, which determines the theta series, denoted by $R \theta_{k}$, is described in the following theorem. 
Theorem 5.2. The vertices of the theta regions $R \theta_{k}$ for $k=48,52$ and 56 are given below (till 2 places of decimal).

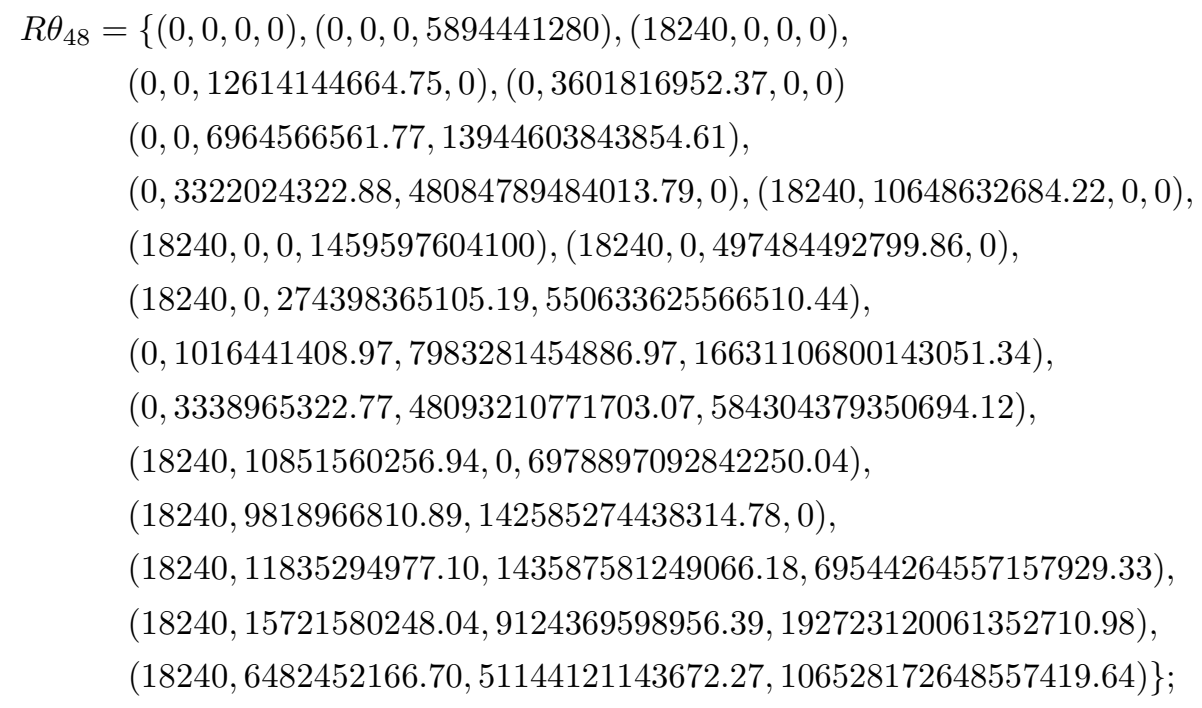


Appendix A. On nORMAlized modular forms of Weights 20, 24, 28 AND 32 WITH NON-NEGATIVE INTEGRAL FOURIER COEFFICIENTS

by

\section{MANICKAM AND B. RAMAKRISHNAN}

In this appendix, we determine exactly the number of modular forms of weight $k$, $(4 \mid k)$, for the group $S L_{2}(\mathbb{Z})$, which behave like theta series for the cases $20 \leq k \leq 32$.

We will first prove the following main lemma.

Lemma A.1. Let $\operatorname{dim} M_{k}(1)=m$. Then we can find a basis $\left\{f_{\ell}(z)=\sum_{n=0}^{\infty} a_{\ell}(n) q^{n}\right.$ $0 \leq \ell \leq m-1\}$ for $M_{k}(1)$ such that $a_{\ell}(n) \in \mathbb{Z}$ for all $n \geq 0,0 \leq \ell \leq m-1$ and $a_{\ell}(j)=\delta_{\ell, j}, 0 \leq \ell, j \leq m-1$, where $\delta_{\ell, j}$ is the Kronecker delta function.

Further, if $f(z)=\sum_{n=0}^{\infty} a_{f}(n) q^{n} \in M_{k}(1)$ is such that $a_{f}(n) \in \mathbb{Z}$ for $0 \leq n \leq$ $m-1$, then $a_{f}(n) \in \mathbb{Z}$ for all $n \geq 0$.

Proof. Let $g_{i}(\tau)=\sum_{n \geq} b_{i}(n) q^{n}(1 \leq i \leq m-1)$ be a basis of $S_{k}(1)$ such that all the $b_{i}(n)$ 's are integers and $b_{i}(j)=\delta_{i, j}$. (See [1, Chapter X, Theorem 4.4].)

Set

$$
g(z)= \begin{cases}E_{4}(z)^{k / 4} & \text { if } k \equiv 0,4,8 \quad(\bmod 12) \\ E_{14}(z) E_{4}(z)^{(k-14) / 4} & \text { if } k \equiv 2 \quad(\bmod 12) \\ E_{6}(z)^{k / 6} & \text { if } k \equiv 6 \quad(\bmod 12) \\ E_{10}(z) E_{4}(z)^{(k-10) / 4} & \text { if } k \equiv 10 \quad(\bmod 12)\end{cases}
$$

Then $g(z)=\sum_{n \geq 0} b(n) q^{n} \in M_{k}(1)$ such that all the $b(n)$ 's are integers. Clearly, $\left\{g, g_{1}, \cdots, g_{m-1}\right\}$ form a basis of $M_{k}(1)$. Now to find the required basis, put

$$
f_{0}(z)=g(z)-\sum_{i=1}^{m-1} b(i) g_{i}(z) \text { and } f_{i}(z)=g_{i}(z) \quad(1 \leq i \leq m-1)
$$

It follows that $\left\{f_{0}, f_{1}, \cdots, f_{m-1}\right\}$ is the required basis of $M_{k}(1)$. The remaining part of the lemma is now clear since $f(z)=\sum_{i=1}^{m-1} a_{f}(i) f_{i}(z)$.

A.1. The case $k=20$. We know that the space $M_{20}(1)$ is spanned by $E_{20}(z)$ and $\Delta(z) E_{4}(z)$. Let $\phi(z)=\sum_{n>0} a(n) q^{n}$ be a modular form in $M_{20}(1)$ which has constant term unity and all other Fourier coefficients are integers; i.e., $a(0)=1$ and $a(1)=X$ (say) with $X(\geq 0) \in \mathbb{Z}$. Then Lemma A.1 implies that all the $a(n)$ 's are integers. $a(n)$ can be written as

$$
a(n)=\frac{13200}{283 \times 617}\left(\sigma_{19}(n)-b(n)\right)+b(n) X
$$

where $\Delta(z) E_{4}(z)=\sum_{n \geq 1} b(n) q^{n}$.

Using the Ramanujan-Petersson estimate (proved by Deligne) for $b(n)$ (i.e., $\left.|b(n)| \leq \sqrt{3} n^{10}\right)$, one can prove from (33) that $a(n) \geq 0$ if and only if $a(4) \geq 0$ and $X \geq 0$. Since $a(4) \geq 0$ and $X \geq 0$ imply $0 \leq X \leq 65686$, we have the following: 
Theorem A.2. The number of modular forms in $M_{20}(1)$ which behave like theta series is 65687 .

A.2. The cases $k=24,28,32$. In these cases $\operatorname{dim} M_{k}(1)=3$. The space $M_{k}(1)$ is spanned by $E_{k}(z), \Delta(z) E_{k-12}(z)$ and $\Delta^{2}(z) E_{k-24}(z)$.

Let

$$
\begin{aligned}
E_{k}(z) & =1+A_{k} \sum_{n=1}^{\infty} \sigma_{k-1}(n) q^{n}, \\
\Delta^{i}(z) E_{k-12 i}(z) & =\sum_{n=i}^{\infty} a_{i, k}(n) q^{n} \quad(i=1,2) .
\end{aligned}
$$

Let $\phi_{k}(z)=1+\sum_{n=1}^{\infty} a_{k}(n) q^{n} \in M_{k}(1)$ be such that

$$
a_{k}(n) \geq 0 ; \quad a_{k}(n) \in \mathbb{Z} \text { for all } n \geq 1 .
$$

Then our problem is to find all $\phi_{k}(z)$ satisfying (35).

Letting $a_{k}(i)=X_{k, i} \in \mathbb{Z}, 1 \leq i \leq 2$, we get

$$
a_{k}(n)=A_{k} B_{k}(n)+\sum_{i=1}^{2} \alpha_{i, k}(n) X_{k, i}
$$

where $\alpha_{1, k}(n)=a_{1, k}(n)-a_{1, k}(2) a_{2, k}(n) \in \mathbb{Z}$ and $\alpha_{2, k}(n)=a_{2, k}(n) \in \mathbb{Z}$ and $B_{k}(n)$ is defined by

$$
B_{k}(n)=\sigma_{k-1}(n)-\sigma_{k-1}(2) a_{2, k}(n)-\alpha_{1, k}(n)
$$

Since $a_{k}(i)=X_{k, i} \in \mathbb{Z}, 1 \leq i \leq 2$, and $a_{k}(0)=1$, from Lemma A.1 we see that $a_{k}(n) \in \mathbb{Z}$ for all $n \geq 0$. This implies that $A_{k} B_{k}(n) \in \mathbb{Z}$.

Using (35) and (36) we get the following bounds for $X_{k, i}$,

$$
0 \leq X_{k, i} \leq I_{k, i}
$$

where $I_{k, i}$ are given in the table below.

\begin{tabular}{|c|c|c|}
\hline $\mathrm{k}$ & $I_{k, 1}$ & $I_{k, 2}$ \\
\hline 24 & 901973 & 3117528477 \\
28 & 3053422 & 22151903688 \\
32 & 12066084 & 151617872159 \\
\hline
\end{tabular}

Let $g_{k}(z) \in S_{k}(1)$ be a normalized Hecke eigenform. Then using the Ramanujan - Petersson estimate proved by Deligne, we have

$$
\left|b_{k}(n)\right| \leq \sqrt{3} n^{k / 2},
$$

where $g_{k}(z)=\sum_{n=1}^{\infty} b_{k}(n) q^{n}$.

Expressing $\Delta(z) E_{k-12}(z)$ and $\Delta^{2}(z) E_{k-24}(z)$ as a linear combination of the basis elements $g_{i, k}(z), 1 \leq i \leq 2$, which are normalized Hecke eigenforms in $S_{k}(1)$, and using (40) we get estimates for the Fourier coefficients $a_{i, k}(n)$ as follows.

$$
\left|a_{i, k}(n)\right| \leq \mu_{k, i} n^{k / 2},
$$


where

\begin{tabular}{|c|c||c|c||c|c|}
\hline $\mathrm{k}$ & 24 & $\mathrm{k}$ & 28 & $\mathrm{k}$ & 32 \\
\hline$\mu_{k, 1}$ & 2.74 & $\mu_{k, 1}$ & 5.57 & $\mu_{k, 1}$ & 3.84 \\
$\mu_{k, 2}$ & $3.8 \times 10^{-4}$ & $\mu_{k, 2}$ & $1.96 \times 10^{-4}$ & $\mu_{k, 2}$ & $3.37 \times 10^{-9}$ \\
\hline
\end{tabular}

Using the bounds from the table (39) and the estimate (41), we can prove that $a_{k}(n) \geq 0$ for all $n$ except for the following cases:

$$
(k, n) \in\{(24,3),(24,5),(24,6) ;(28,4),(28,7),(28,8) ;(32,5),(32,9),(32,10)\} .
$$

We have thus the following theorem.

Theorem A.3. $a_{k}(n) \geq 0$ if and only if $a_{k}\left(i_{k}\right) \geq 0$ where

$$
i_{k}= \begin{cases}1,2,3,5,6 & \text { if } k=24 \\ 1,2,4,7,8 & \text { if } k=28 \\ 1,2,5,9,10 & \text { if } k=32\end{cases}
$$

The following theorem is obtained using the Simplex method for solving a Linear Programming problem following the present work in the paper.

Theorem A.4. $a_{k}\left(i_{k}\right) \geq 0$ and $a_{k}\left(i_{k}\right) \in \mathbb{Z}$, where $i_{k}$ is as in Theorem A.3, if and only if $X_{k, i} \quad(1 \leq i \leq 2)$ belong to the following convex region, denoted by $R_{k}$, in $\mathbb{R}^{2}$ given by the following vertices:

$$
\begin{aligned}
R_{24}=\{(0,0),(0,1092000),(395935.3361,1615023413.7759), & (901973.05768614149406,3117528477.5604),(445623.6915,0)\} ; \\
R_{28}=\{(0,0),(0,4463440.4348),(3004644.4353,22151903688.7315), & (3053422.7929,5108328206.416),(2378265.7383,0)\} ; \\
R_{32}= & \{(0,0),(0,13898141.4518),(11533050.8143,151617872159.7915), \\
& (12066084.8402,107182839332.9432),(8056296.7887,0)\} .
\end{aligned}
$$

In the above, the vertices are given till 4 places of decimal.

Remark A.1. Noticing that to each lattice point in the region $R_{k}$ one gets a modular form which behaves like a theta series, the number of modular forms in $M_{k}(1)$ which behave like theta series, denoted by $N(k)$, can be obtained and we give below the numbers.

$$
\begin{aligned}
& N(24)=806022416212942, \\
& N(28)=32642069239095156 \\
& N(32)=566165632956673500
\end{aligned}
$$

Remark A.2. A view of the convex region $R_{24}$ ( $X$ coordinates scaled down by 100 and the $Y$ coordinates scaled in logarithms for the nonzero entries) is given in Figure A. 


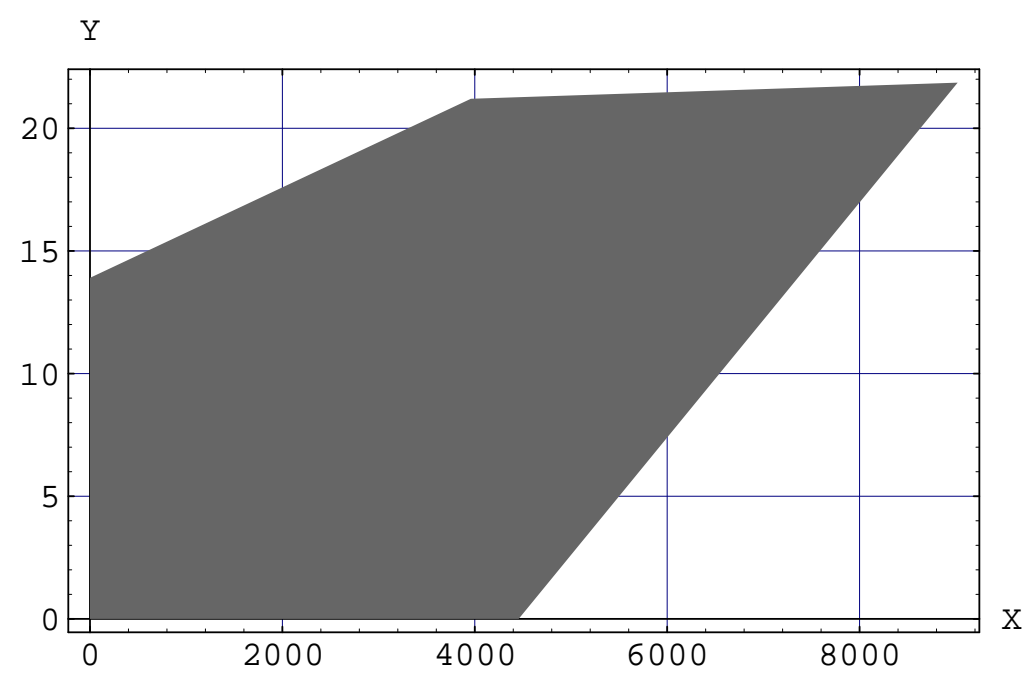

Figure A

\section{REFERENCES}

1. S. Lang, Introduction to Modular Forms, Springer-Verlag, New York 1976. MR 55:2751

2. M. Manickam, Newforms of half-integral weight and some problems on modular forms, $\mathrm{Ph}$. D. Thesis, Univ. of Madras 1989.

3. M. Manickam and B. Ramakrishnan, On normalized modular forms of weights 20,24 and 28 with non-negative integral Fourier coefficients, preprint 1985.

4. A. M. Odlyzko and N. J. A. Sloane, On exceptions of integral quadratic forms, J. reine angew Math. 321, 212-216 (1981). MR 82b:10025

5. M. Ozeki, On modular forms whose Fourier coefficients are non-negative integers with the constant term unity, Math. Ann. 206, 187-203 (1973). MR 48:8392

6. J. -P. Serre, A Course in Arithmetic, Springer-Verlag, New York 1973. MR 49:8956

Mehta Research Institute of Mathematics and Mathematical Physics, 10, Kasturba Gandhi Marg (Old Kutchery Road), Allahabad 211 002, India

E-mail address: kalyan@mri.ernet.in

Current address, A. K. Lal: Department of Mathematics, Indian Institute of Technology, Kanpur 208 016, India

E-mail address: arlal@iitk.ernet.in

E-mail address: ramki@mri.ernet.in 